\section{P204 Relationship Between Dietetic Students' Travel and Study Abroad Experience and Cultural Competence}

Tanya M. Horacek, PhD, RD, thoracek@syr.edu, Syracuse University, 558 White Hall, Syracuse, NY 13244

Objective: Given the demographics and health disparities in the U.S., there is a greater need for culturally competent healthcare providers. Cultural competence research for nursing and physical therapy students is extensive. This study investigates dietetic students' cultural competence.

Study Design, Setting, Participants: Dietetic students participated in an online anonymous survey about their multicultural personality (MP) and travel and study aboard experiences.

Outcome Measures and Analysis: MP Questionnaire uses a 5-point scale to evaluate adaptability to other cultures via five personality traits: cultural empathy, flexibility, social initiative, open-mindedness, and emotional stability. The Multicultural Experiences Questionnaire uses a 5-point Likert scale to measure cultural experiences/attitudes. Nonparametric statistics, t-tests and ANOVA were used.

Results: Of the 220 students who participated, 19\% had never traveled outside of the United States. Most (59\%) students had participated in at least one course related to intercultural communication. There were a few significant difference by travel experience $(P<0.05)$, students whom traveled out of the country, 1-2 times in their lifetime were less open to media and art from other countries; whereas those with the most travel experience were significantly more likely to seek people of different backgrounds. Only 20\% of the students, had already studied abroad, and $20 \%$ intend to in the near future. Conclusions and Implications: Because of the structure and specificity of dietetic curricula, most students lack the option of traveling abroad for an entire semester, which makes short term aboard experiences a crucial opportunity for students to enhance their cultural competency. The results of this survey call for a further analysis of dietetic students and the effect of short-term study abroad programs on their cultural competencies.

Funding: Syracuse University

\section{P205 The Johns Hopkins School of Medicine (JHSOM) Teaching Kitchen: A Novel Recipe for Nutrition Education}

ShannonWongvibulsin, BS, swongvi1@jhmi.edu, Johns Hopkins School of Medicine, 1830 East Monument Street, Suite 2-300, Baltimore, MD 21205; Shivani Bhat, MPH, BSC, Global Centre for Nutrition and Health;

Sumantra Ray, MD, MPhil

Objective: To develop and implement an interactive educational model to equip medical students with the skills to address the increasing prevalence of nutrition-related diseases.

Theory, Prior Research, Rationale: Although the public generally considers doctors as "very credible" sources of nutrition information, nutrition is generally underemphasized in medical school curricula and most students do not feel adequately trained in nutritional counseling. To address the deficiency of nutrition education in the medical profession, "Teaching Kitchens" have emerged as interactive, hands-on methods for nutrition education. The conceptual framework behind the "Teaching Kitchen" model is based on the general goals of providing nutrition knowledge that enables physicians to counsel patients on nutrition-related issues and serve as role models of healthy dietary practices.

Description: The JHSOM Teaching Kitchen is unique in that it was student-initiated and founded by first year medical students who recruited faculty mentors as well as registered dietitians and culinary experts. This multidisciplinary team with medical, nutrition, and culinary expertise have worked together to develop an engaging curriculum to convey essential nutrition knowledge and culinary skills that students can use to counsel patients on the impact of food and diet on their health.

Evaluation: Pre and post-surveys are administered to evaluate the impact of Teaching Kitchen sessions on culinary skills and nutrition knowledge and determine improvement strategies for future sessions.

Conclusions and Implications: The "Teaching Kitchen" model provides a multidisciplinary and innovative platform for medical students to gain nutrition knowledge and practical culinary skills. Moreover, this model is a promising method to equip the medical profession with the knowledge and skills necessary to address the global increase in nutrition-related diseases.

Funding: None

\section{P206 Thrifty Meal Plan Survivor Challenge: A Powerful Simulation for Nutrition Interns}

Kathy Savoie, MS, RD, ksavoie@maine.edu, UMaine Extension, 75 Clearwater Drive, Suite 104, Falmouth, ME 04105

Objective: To prepare future nutrition professionals for work with food insecure populations through a simulation experience sensitizing interns to the reality of living on a limited food budget.

Theory, Prior Research, Rationale: Simulations are a type of interactive educational exercise that promotes experiential learning as learners live through a 'real-life' situation. Simulations are an effective learning method that impacts retention of knowledge and attitude change.

Description: Target Audience was second year Dietetic Technology students enrolled in Community Nutrition Internship $(\mathrm{n}=40)$. The Thrifty Meal Plan Survivor Challenge (TMPSC) is a simulation exercise that immerses interns in a 'real-life' situation to plan, shop and prepare a week's worth of meals and snacks according to the Thrifty Food Plan Market Basket Standards while remaining below the Cost of Food at Home economical confines for the Thrifty Meal Plan. While completing the TMPSC, interns are encouraged to adopt 'real-life' situations of food insecure populations (ex: limited transportation, access and resources).

Evaluation: The results of the TMPSC six reflection prompts indicated an overall increase in understanding

Continued on page $\mathrm{S} 105$ 